\title{
Efeitos de instrução e presença de selo na escolha entre produtos sustentáveis e não sustentáveis
}

\author{
The effects of instructions and labels on choice between environmental \\ friendly and non-friendly products.
}

\section{Efectos de instrucción y presencia de sello en la elección entre productos sostenibles y no sostenibles}

\author{
Reginaldo Pedroso ${ }^{1}$, Cristiano Coelho ${ }^{2}$
}

Resumo: O presente estudo visou comparar o efeito de instruções e presença do selo de certificação ambiental sobre escolhas entre produtos com e sem sinalização de produção sustentável em dois experimentos: 1) preço de diferentes produtos (lâmpada, amaciante, papel sulfite e cama) com selos ambientais relativamente a produtos sem selo ambiental, com e sem instrução pró-ambiental, com 24 universitários; 2) diversas marcas de um mesmo produto (amaciante), com e sem selo ambiental, visando estimar se o preço relativo variaria em função do nível de conhecimento, qualidade e sustentabilidade, com 12 universitários. A tarefa em ambos os experimentos era escolher um produto entre cada par em escolhas sucessivas. No Experimento 1, os dados demonstraram que apenas para lâmpada os preços subjetivos relativos com as duas instruções foram maiores para o produto sustentável. No Experimento 2, observou-se que quanto maior o nível de conhecimento e qualidade menor o preço relativo ao produto não sustentável. A ausência de relação entre preço relativo e sustentabilidade mostra que o selo teve baixa propriedade discriminativa. Uma das possibilidades de intervir para aumentar os comportamentos pró-ambientais envolve um conjunto de contingências para o indivíduo e para as empresas que produzem.

Palavras-chave: desenvolvimento sustentável, análise do comportamento, consumo sustentável, escolha, efeito de instrução 
Abstract: This study aimed to compare the effect of instructions and the presence of the environmental certification on choices between products with and without signal of sustainable production in two experiments: 1) Price of different products (lamp, softener, sulphite paper, and bed) with environmental certifications relative to products without environmental certification, with and without pro-environmental instruction, with 24 undergraduate students; 2) Several brands of the same product (softener), with and without environmental certification, aiming to estimate if the relative price would vary according to the level of knowledge, quality and sustainability, with 12 undergraduate students. The task in both experiments was to choose one product between each pair in successive choices. In the Experiment 1, the data showed that only for the lamp the relative subjective prices with the two instructions were higher for the sustainable product. In the Experiment 2, it was observed that the higher the level of knowledge, the lower the price relative to the non-sustainable product. The absence of correlation between relative price and sustainability shows that the certification had low discriminative properties. One of the possibilities for intervening to increase pro-environmental behavior involves a set of contingencies for the individual and the companies that produce them.

Keywords: sustainable development, behavior analysis, sustainable consumption, choice, instructional effect.

Resumen: El presente estudio pretendió comparar el efecto de las instrucciones y la presencia del sello de certificación ambiental sobre la elección entre diferentes productos (lámpara, suavizante, papel A4 y cama), con y sin señalización de producto sostenible en dos experimentos: 1) precio de diferentes productos con sellos ambientales en relación a productos sin sello ambiental, con y sin instrucción pro ambiental, con 24 universitarios, 2) distintas marcas de un mismo producto (suavizante) con y sin sello ambiental, visándose estimar el precio relativo en función del nivel de conocimiento, cualidad y sostenibilidad, con 12 universitarios. La tarea en ambas fases era elegir un producto entre cada par en elecciones sucesivas. En el Experimento 1 los datos demuestran que sólo con lámparas los precios subjetivos relativos con las dos instrucciones fueron mayores para el producto sostenible. En el Experimento 2 se observó que cuanto mayor el nivel de conocimiento y calidad, menor fue el precio subjetivo relativo al producto no sostenible. La ausencia de relación entre precio relativo y sostenibilidad muestra que el sello tuvo baja propiedad discriminativa. Una de las posibilidades de intervenir para aumentar los comportamientos pro-ambientales implica un conjunto de contingencias para el individuo y para las empresas que producen.

Palabras-clave: desenvolvimiento sustentable, análisis de la conduta, consumo sostenible, elección, efecto de instrucción. 
É notório que a relação entre estilo de vida e práticas de consumo vem resultando em graves problemas ambientais, por exemplo: mudanças climáticas, enchentes, secas prolongadas, extinção de espécies de animais e plantas. A mudança no atual padrão de consumo tornou-se uma questão de sobrevivência (Furtado, 1974, 2009; Sachs, 2009). A compreensão do comportamento do consumidor e sua relação com produtos sustentáveis pode ser uma alternativa importante para o direcionamento de novas estratégias que gere uma prática de consumo consciente (De Toni, Mattia, \& Larentis, 2012). ), ou seja, que pessoas passem a escolherem produtos e serviços que agridem menos o meio ambiente.

Os estudos em geral (e. g., Marketing, Administração, Ciências Sociais), usam o termo consumidor consciente na busca de entender como esse consumidor vem se relacionando com as questões ambientais (Fabi, Lourenço, \& Silva, 2010; Gomes, 2006; Rodrigues, Gonçalves, Costa, Nora, \& Rezende, 2011; Silva \& Gomes, 2010). Nesse contexto, consumo consciente seria o modo de vida no qual se valoriza a responsabilidade ambiental como maneira de preservar e manter um meio social com maior qualidade de vida (De Toni, Mattia, \& Larentis, 2012).

No âmbito da análise do comportamento, os estudos buscam identificar o controle de variáveis situacionais sobre a escolha de produtos. À medida que o comportamento entra em contato com suas consequências, ele passa a ficar sob controle de estímulos discriminativos e, em ocasiões futuras, esses estímulos passam a sinalizar a probabilidade de reforço ou punição para a ocorrência de um dado comportamento (Skinner, 2003/1953). No contexto de consumo, as marcas são estímulos que sinalizam a probabilidade de reforço para a compra, uso ou descarte de um produto. Tendo em vista que as consequências exercem controle sobre o comportamento, no caso do coportamento do consumidor, este pode ser reforçado pelos benefícios da compra de um produto ou serviço (reforço utilitário) ou pode ser reforçado pela aprovação social (reforço informativo), como também é simultaneamente punido (consequências aversivas), fato de ter que dispensar de reforçadores condicionados generalizados (punição utilitária) ou feedback social negativo (punição informativa), por exemplo
(Foxall, Oliveria-Castro, James, Yani-de-Soriano \& Sigurdsson, 2006).

Um grande número de marcas disponíveis para o consumidor traz atributos diferenciados para a escolha de um produto (Nalini, Cardoso \& Cunha, 2013). Foxall, Oliveira-Castro e Schrezenmaier (2004) demonstram que escolhas de marcas são, em parte, função do nível de reforço informativo e utilitário programados pela marca. A escolha do consumidor também sofre influência de benefícios criados por estratégias de Marketing, sendo seus efeitos inerentes à marca (Foxall, James, OliveiraCastro \& Ribier, 2010). Atualmente, pesquisadores da área de Marketing vêm desenvolvendo estratégias no intuito de levar o consumidor escolher produtos sustentáveis (Botelho \& Manolescu, 2010; Gonzaga, 2005; Kohlrausch, 2003; Tomé, 2008). No entanto, qualquer que seja a estrátegia para modificar o comportamento do consumidor que produz efeitos indesejaveis sobre o meio ambiente deve primeiro identificar de quais variaveis o comportamento é função (Foxall et al., 2006).

$\mathrm{O}$ uso de selos em determinadas marcas (selos sustentáveis, verdes, ecológicos, entre outras nomenclaturas) foi uma das estratégias do Marketing para colocar o consumidor sob controle de parte do cenário de compra, aumentando a probabilidade de escolhas por produtos que geram menos danos ao meio ambiente. Esses selos são etiquetas colocadas em produtos com a finalidade de tornar a marca diferenciada no mercado, sinalizando que uma empresa se preocupa, em alguma parte do seu ciclo de produção, com as questões ambientais, (Deus, Felizola, \& Silva, 2010; Dias, 2008; Godoy \& Biazin, 2000; Tomé, 2008). Segundo Tomé (2008), “Um consumidor/comprador, entre decidir adquirir um produto ou serviço ao qual tenha sido concedida uma ecoetiqueta e outro produto ou serviço convencional, sem qualificação extra, tenderá a decidir pelo produto ecoetiquetado" (p. 37).

No entanto, estudos que verificaram o efeito do selo ambiental sobre a escolha por produtos sustentáveis demonstram que essa estratégia, mesmo com o crescente número de produtos ecoetiquetados e uma ampla variedade de selos, tem pouco efeito sobre o comportamento do consumidor em escolher produtos sustentáveis (Barbosa, Araujo, \& Cabral, 2014; Campos, Grzebeiluckas, \& Selig, 
2009; Castilho \& Gómez, 2014; Castro, Paracampo \& Albuquerque, 2015; Kohlrausch, Campos, \& Selig, 2004; Valent, Vieira, Bruzza et al., 2014; Veras, Souza, Araújo et. al., 2014).

No mercado, esses produtos tidos como sustentáveis têm preços superiores em relação aos produtos substitutos (Foxall, et. al., 2006), visto que os produtos sustentáveis se tornam mais caros devido ao custo para sua fabricação. Segundo a Carta da Terra (The Earth Charter Initiative, 2003, p. 4), para que sejam atingidos os princípios de responsabilidade sustentável é necessário "Incluir totalmente os custos ambientais e sociais de bens e serviços no preço de venda e habilitar os consumidores a identificar produtos que satisfaçam as mais altas normas sociais e ambientais". No entanto, o consumidor tende, em diferentes ocasiões, a comprar quantidade menor de um produto quando este tem preços mais elevados. Esse padrão de consumo pode estar relacionado às caracteristicas demográficas, tais como o tamanho da família e renda, o que pode determinar a quantidade que consumidores precisam comprar e os preços que eles podem pagar (Cavalcanti, Oliveira-Castro, \& Foxall, 2013; Oliveira-Castro, Foxall, \& Schrezenmaier, 2006; Oliveira-Castro, Foxall, \& James, 2008; OliveiraCastro, Foxall, \& Wells, 2010).

A questão do preço do produto sobre o comportamento do consumidor, fundamental na análise de comportamentos pró-ambientais, nem sempre é uma situação na qual as empresas têm controle. Os preços de um produto dependem muito mais da dinâmica do próprio mercado (Furtado, 2009), por exemplo a Lei da oferta e procura (Ulbra, 2008). Isso justifica uma avaliação das condições relacionadas à escolha, incluindo-se o preço. Neste campo as pesquisas têm utilizado procedimentos nos quais os participantes indicam o preço estimado de variáveis ambientais e/ou respostas a questionários ou surveys, para os quais são analisadas as possíveis relações e tomadas como indicativo de intenção de consumo pró-ambiental (Braga, Viana, Abreu \& Faria, 2008; Macedo, 2009; Romeiro \& Maia, 2011). Contudo, estudos demonstram que em alguns casos os consumidores estariam aptos a pagarem mais por um produto sustentável, em outros não.

Gorni, Gomes e Dreher (2012), ao analisaram o comportamento, discurso e prática, de 312 univer- sitários sobre o consumo sustentável. Para a coleta de dados foram utilizadas as escalas Environmental Concern (consciência ambiental) e Ecologically Conscious Consumer Behavior (atitudes pró-ambientais). Os resultados demonstraram que os discursos dos homens e mulheres divergiram das afirmativas em relação às práticas de consumo sustentável e a consciência ambiental, mas para ambos o preço foi o fator mais considerado na escolha de um produto.

Félix e Nunes (2014) investigaram se após sensibilização, consumidores estariam dispostos a mudar o produto que consomem por produtos com argumentos sociais e ambientais. O produto escolhido foi o sabão em pó, pertencente a uma marca que se posiciona no mercado como livre de fosfato na fórmula. Os participantes receberam uma amostra do produto de uma Marca livre de fosfato em suas residências e responderam a um questionário comparando o produto teste com outras marcas de sabão em pó, e responderam a um questionário. Os 41 questionários devolvidos, todos de participantes de classe econômica $\mathrm{A}$ e $\mathrm{B}$, demonstraram que dentre os seis atributos pesquisados, para quatro a semelhança entre a marca enviada e as demais marcas dos produtos foi superior a $90 \%$. Para os outros dois atributos, capacidade espumante e rendimento, a semelhança foi de $64 \%$ e $59 \%$, respectivamente. Para os autores, esses dados demonstram elevado potencial de substituição entre os produtos.

A segunda fase do estudo supracitado, os pesquisadores propuseram verificar se os participantes trocariam pelo produto com apelo à sustentabilidade. Para a pergunta "eu estaria disposto a trocar a marca de sabão em pó que compro habitualmente por esta ora testada, se soubesse tratar-se de um produto ambientalmente mais correto", $89 \%$ dos consumidores mostraram dispostos a substituir a marca. Com a pergunta, "caso este sabão tenha o mesmo preço que a marca que uso hoje, sinto-me com a seguinte sensação sobre passar a usá-lo", 78\% dos participantes demonstraram dispostos a mudar de produto. Quando perguntados, "ao preço de R\$ 5,00 que marca você prefere?", 55\% dos participantes relataram preferir a marca livre de fosfato. Quando não era sugerido o preço, $78 \%$ optaram pela marca livre de fosfato. Na tentativa de investigar a disponibilidade a pagar pelo produto, foi realizada uma 
relação aos produtos Marca livre de fosfato e Marca Top. Foram sugeridos ao consumidor nove preços distintos de $\mathrm{R} \$ 4,00$ a $\mathrm{R} \$ 7,00$. Os dados demonstram um preço modal de $\mathrm{R} \$ 5,20$ para os dois produtos, uma propensão a pagar pelo produto com características de sustentabilidade o mesmo preço do produto mais vendido no mercado.

Back, Hahn e Scherer (2015) utilizaram uma pesquisa survey com objetivo de identificar a consciência ambiental e as atitudes de consumo sustentável de indivíduos no âmbito internacional. Os pesquisadores analisaram 78 questionários recebidos de 26 países. Foram criados dois construtos, um sobre consciência ambiental e outro sobre consumo sustentável. Os resultados demonstram que os respondentes se mostraram indiferentes em relação à sua preocupação na compra e consumo de produtos ambientalmente corretos, mas, em resposta à pergunta "quando se conhece os danos que um produto pode causar ao meio ambiente, eu não compro o produto", 67,9\% demonstraram conscientes. Também, a amostra indicou uma pré-disposição a pagar um pouco mais por produtos e alimentos que estão livres de elementos químicos e que prejudicam o meio ambiente. Para consumo de energia, os respondentes não têm hábitos de usar eletrodomésticos fora do horário de pico de consumo, mas se preocupam em economizar energia comprando lâmpadas mais caras e eletrodomésticos que consomem menos. Especificamente sobre o subconstruto da consciência ambiental, os dados demonstraram, no geral, que os participantes foram indiferentes e, segundo os autores, pode-se inferir que as pessoas respeitam o meio ambiente, desde que os meios utilizados para isso não afetem seu próprio conforto e bem-estar.

Pinto e Pedroso (2015) verificaram em uma amostra de 80 consumidores o quanto estariam dispostos a pagar por um produto visto como sustentável em uma parte de seu ciclo de vida. Foi utilizado como o produto cerveja long neck. Os participantes foram divididos em dois grupos, com e sem instrução, e dois subgrupos, masculino e feminino. Para o grupo sem instrução, era perguntado se o casco da cerveja fosse retornável o quanto estaria disposto a pagar pela cerveja, que custava $\mathrm{R} \$ 8,00$. Para o grupo com instrução, antes da pergunta sobre o preço a pagar era apresentado um texto descrevendo os atuais problemas ambientais e o tempo para que o vidro fosse absorvido na natureza. Os resultados demonstraram que a maioria dos participantes pagaria menos que o valor real de $\mathrm{R} \$ 8,00$. No grupo sem instrução, nenhum participante se propôs a pagar mais pelo produto; para o grupo com instrução, quatro participantes pagariam a mais. Ou seja, os dados demonstraram que, de acordo com essa amostra e para esse produto, consumidores tenderam a não gastar mais por um produto sustentável.

A discrepância nos resultados dos estudos supracitados faz-se questionar o tipo de metodologia utilizada para a coleta de dados. O que se percebe nos dados supracitados, é que quando há disposição a pagar mais, o valor não pode ser muito diferente e também não pode afetar o bem-estar. A discussão sobre o uso de questionários na coleta de dados não é nova, e nem tem sinal de encontrar um consenso. A área de estudo sobre correspondência entre dizer e fazer demonstra que nem sempre o que a pessoa diz fazer ela faz, e nem sempre o que ela faz, ela diz (e.g. Braga, Albuquerque, \& Paracampo, 2005; Simonassi, 1999). Nesse sentido, o uso de questionários pode trazer análises com vieses que não correspondem à realidade. As divergências encontradas em estudos sobre Disposição a Pagar podem ser justificadas pelo uso dessa metodologia em suas coletas de dados. Sendo que para a coleta de dados, é comum a utilização de mercado hipotético e análises estatísticas e as solicitações de preço são feitas de forma direta, sem criar situações nas quais os indivíduos têm que escolher diretamente entre dois produtos. Além disso, estudos que vêm utilizando a média de conhecimento e qualidade (MCQ) de produtos e marcas, na análise do comportamento do consumidor na Perspectiva Comportamental (BPM - Behavioural Perspective Model), estabelece uma forte correlação entre conhecimento, qualidade o consumo observado (Dias \& Oliveira-Castro, 2006; Faustino, 2009; Pohl \& Oliveira-Castro, 2004).

Comportamento de escolha vem sendo estudado, na perspectiva analítico-comportamental, prioritariamente sob o rótulo da Lei de Igualação, a qual foi derivada de estudos experimentais que propõem que a distribuição do comportamento é função da distribuição dos reforços disponíveis 
em duas fontes (Baum, 1974; Herrnstein, 1961, 1970). A partir da igualação desenvolveram-se, por exemplo, os estudos sobre o efeito de atraso e probabilidade sobre o comportamento de escolha (Coelho, Hanna, \& Todorov, 2003; Mazur, 1988; Rachlin, Ranieri, \& Cross, 1991).

As manipulações em atrasos e probabilidade para o recebimento de uma quantia em relação à outra quantia menor, mas imediata, permite que se avalie o valor imediato ou certo de uma quantia atrasada ou provável, conforme o ponto de indiferença1, que permite observar mudanças em tomadas de decisão à medida que seu atraso ou probabilidade variam. Essa medida permite que seja estimado o desconto de uma dada quantia, ou seja, como ela perde valor em função do atraso ou probabilidade para o recebimento da mesma (Todorov, 2005).

Pesquisas sobre desconto têm seguido uma linha de pesquisas empírica com formulação de modelos matemáticos para os fenômenos comportamentais, e esses modelos vêm demonstrando fortes preditores para desconto de consequências atrasadas e incertas (Hirsh, Costello \& Fuqua, 2015). Além disso, pesquisas na área de escolha e tomada de decisão vem utilizando manipulação na forma que a instrução é apresentada e seu efeito sobre a preferência e julgamentos (Coelho et al., 2003; Tversky \& Kahneman, 1981), mostrando que em determinados contextos a forma de apresentação da instrução (frame) influenciaria tomadas de decisões em situações hipotéticas de risco. Para Tversky \& Kahneman (1981), a forma de apresentação da instrução altera o referencial a partir do qual os indivíduos fazem seus julgamentos ou realizam suas decisões.

Situações de escolha entre o consumo de um produto sustentável e um produto não sustentável envolvem consequências de longo prazo para o grupo (manutenção das condições de produção, qualidade de vida no longo prazo, etc.) e consequências imediatas para o indivíduo (menor preço a

1 Ponto de indiferença ou valor subjetivo é um termo utilizado em estudos sobre comportamento de escolha em situação de risco, o qual é subtraído a partir do ponto de mudança da escolha pela quantia incerta/atrasada para a quantia certa/ imediata (Rachlin et al., 1991) pagar, qualidade do produto ou serviço). Contudo, por vezes, os indivíduos não estabelecem relações entre seus comportamentos (como comprar um produto que degrada o meio ambiente, seja na produção, seja no uso ou no descarte) e consequências de longo-prazo (como a degradação ambiental e poluição de mananciais).

Uma das possibilidades de intervir para aumentar os comportamentos pró-ambientais envolve um conjunto de contingências para o indivíduo e para as empresas, como subsídios para produções sustentáveis e coleta seletiva para produtos recicláveis, as quais dependem de ações do poder público. Em situações nas quais não temos acesso à emissão do comportamento diretamente, uma possibilidade é a utilização de instruções ou regras que especifiquem consequências da produção não sustentável e da produção sustentável.

A partir dessas considerações, o presente estudo visou comparar o efeito de instruções e presença do selo de certificação sobre a escolha hipotética entre produtos com e sem sinalização de produção sustentável. Mais especificamente, o presente estudo foi composto de dois experimentos: no Experimento 1, verificou-se se o preço relativo de produtos de diferentes categorias com selos ambientais relativamente a produtos sem selo ambiental e se uma instrução pró-ambiental alteraria esse preço relativo; no Experimento 2, diversas marcas de um mesmo produto, com e sem selo ambiental foram apresentadas em pares de escolha, visando-se estimar o efeito entre o nível de conhecimento, qualidade e sustentabilidade sobre o preço relativo.

\section{Método}

\section{Experimento 1}

\section{Participantes}

Participaram deste experimento 24 pessoas de ambos os sexos, sendo sete do sexo masculino e 17 do sexo feminino, responsáveis por compras em suas residências, sendo 12 funcionários administrativos de uma IES e 12 estudantes de pós-graduação de diferentes cursos de, com idades de 23 a 60 anos. 


\section{Materiais}

Foram utilizados protocolos de respostas de escolha com fotos de duas marcas de papel sulfite, duas marcas de amaciante de roupas, dois tipos de lâmpadas e dois tipos de camas. O protocolo de coleta continha cinco folhas. Na primeira eram apresentadas as instruções e em cada uma das folhas seguintes eram apresentadas as fotos de um produto sustentável e um produto não sustentável e os pares de escolha com os valores de um dos produtos sustentável permanecendo fixo e do outro produto variando. As escolhas eram entre uma marca com selo de certificação ambiental (Selo SOS Mata Atlântica para amaciante de roupas, Selo ISO 14025 para o papel sulfite reciclado e selo Cer Flor e selo Inmetro para papel sulfite não reciclado, Energy saver para lâmpada e Símbolo de reciclável para cama) e uma marca sem selo ou indicação de ação ambiental.

\section{Procedimento}

Para cada marca dos produtos foram obtidos os preços praticados e tomou-se o valor arredondado mais próximo do preço médio para definição do preço-base de uma das marcas ( $\mathrm{R} \$ 10,00$ para amaciante, $\mathrm{R} \$ 5,00$ para lâmpada, $\mathrm{R} \$ 500,00$ para cama e R $\$ 25,00$ para papel sulfite). Esses preços foram alocados para uma marca com selo de sustentabilidade e permaneceram fixos ao longo do experimento. Para as outras marcas, os preços variaram de $\mathrm{R} \$ 5,00$ a $\mathrm{R} \$ 15,00$ para amaciante, de $\mathrm{R} \$ 3,00$ a $\mathrm{R} \$ 15,00$ para lâmpada, de $\mathrm{R} \$ 100,00$ a $\mathrm{R} \$ 1.000,00$ para cama e de $\mathrm{R} \$ 5,00$ a $\mathrm{R} \$ 50,00$ para papel sulfite. Durante a aplicação, os participantes foram convidados nos respectivos locais de trabalho ou sala de aula para responderem a um questionário sobre comportamento do consumidor. Após lerem e assinarem o Termo de Consentimento Livre e Esclarecido, os participantes receberam o caderno com os protocolos de escolha que apresentavam os pares de marcas dos diferentes produtos.

Foram utilizadas duas instruções, apresentadas cada uma a um conjunto de 12 participantes, uma genérica e uma que acrescentava frases enfatizando os efeitos da degradação do planeta e o impacto ambiental da ação humana.

O planeta clama por cuidados. Nos últimos anos o impacto da ação do homem sobre o meio ambiente gerou mudanças irreparáveis. Boa parte desse impacto está associada ao tipo de consumo da população. Estamos consumindo cada vez mais, e para alcançarmos a sustentabilidade do planeta será necessária uma mudança no estilo de vida, ou seja, mudar do ter mais para ter melhor, e do consumir para o de usufruir. Para que isso ocorra, uma mudança cultural é prioritária para que novos valores e comportamentos emerjam na sociedade.

Algumas empresas já demonstram preocupação com o futuro do planeta. Produtos mais econômicos, com utilização de material reciclado, investimento em reflorestamento e produtos que produzem menos resíduos já estão disponíveis no mercado.

Mas nem todas as empresas mudaram suas práticas buscando a sustentabilidade do planeta. Encontra-se disponível no mercado uma variedade de produtos a disposição dos consumidores, alguns produtos gerando menos impacto sobre o meio ambiente e outros produtos gerando mais impacto. $\mathrm{O}$ que diferenciam esses produtos são selos ambientais que sinalizam quais produtos são considerados sustentáveis. Cabe ao consumidor a outra parte: escolher produtos com selo sustentável diminuirá o impacto sobre o meio ambiente. Preocupar hoje com a sustentabilidade proporcionará um planeta melhor para gerações futuras.

(A parte acima era apresentada apenas para os participantes do grupo com ênfase ambiental, acrescentada da instrução genérica abaixo, apresentada para o outro grupo)

Suponha que você saiu de casa para comprar alguns produtos como amaciante, lâmpada, papel A4 e uma cama, produtos que estão faltando em sua casa. Chegando ao supermercado você se depara com uma variedade de marcas de um mesmo produto. Sua tarefa será escolher entre dois produtos, o de sua preferência. No par de produtos terá um selo que identifica qual produto é sustentável, mudanças no preço ocorrerão a cada escolha conforme a mudança de preço entre os dois produtos você deverá escolher um, marcando com um $\mathrm{X}$ ao lado da figura do produto escolhido. Observe atentamente os valores apresentados e faça uma nova escolha. Lembre-se que a cada mudança na tela é uma nova escolha, independente das anteriores com novos preços. Em cada vez que forem apresentadas novas opções, tente escolher o mais próximo possível ao 
que escolheria se estivesse realizando a compra.

Se você tiver alguma dúvida, pergunte ao pesquisador. Ele ficará a sua disposição.

Ao final, chame o pesquisador.

Desde já agradecemos por sua participação.

Aos participantes, era apresentado para cada produto um conjunto de pares de escolha, com preço médio conforme estimado nos supermercados ou no site de venda mantido constante para uma das marcas e o preço da outra marca variando em sucessivas escolhas. $\mathrm{O}$ número de escolhas foi de 11 para amaciante, 10 para papel sulfite e cama, e de 13 para lâmpada. Para amaciante, o preço da marca sustentável ficou fixo e o da marca não sustentável variou nos sucessivos pares; para os demais produtos, o preço da marca sustentável variou e da marca não sustentável permaneceu fixo nas tentativas sucessivas. Os participantes tinham que indicar sua escolha para todos os pares de preços apresentados.

\section{Experimento 2}

\section{Participantes}

Participaram deste experimento 14 pessoas de ambos os sexos, sendo oito do sexo masculino e seis do sexo feminino, responsáveis por compras em suas residências. Dos participantes, três eram funcionários administrativos de uma IES e 11 estudantes de pós-graduação de diferentes cursos, com idades de 22 a 57 anos (média de 35,9 anos).

\section{Materiais}

Foram utilizados protocolos de respostas de escolha com fotos de quatro marcas de amaciante de roupas: Comfort, Babysoft , Monbijou e Ypê, sendo que Ypê apresentava o selo IBD de certificação ambiental, e uma foto alterada da marca Comfort com a inserção digital do selo IBD de certificação ambiental no local do código de barras. Cada um de cinco protocolos apresentava escolha entre o amaciante Comfort com o selo de sustentabilidade inserido e uma das marcas, gerando escolhas entre os pares Comfort_Sustentável $\mathrm{x}$ Comfort_Original, Comfort_Sustentável x Monbijou, Comfort_Sustentável x Ypê_Sustentável e Comfort_Sustentável x Babysoft.

\section{Procedimento}

Durante a aplicação, os participantes foram convidados nos respectivos locais de trabalho ou sala de aula para responderem a um questionário sobre comportamento do consumidor. Após lerem e assinarem o Termo de Consentimento Livre e Esclarecido, os participantes receberam o caderno com os protocolos de escolha que apresentavam os pares de marcas de amaciantes. Foi utilizada uma instrução genérica que indicava aos participantes para se imaginarem realizando compra deste produto e informada a existência de marcas de amaciantes que têm certificação ambiental por investir no meio ambiente e de marcas que não possuem certificação, conforme a instrução abaixo:

Suponha que você saiu de casa para comprar um amaciante. Para cada tipo você tem à sua disposição dois produtos de uma mesma marca, ou de marcas diferentes. A empresa vende um produto que parte de suas vendas é investida no meio ambiente e por isso tem um selo ambiental estampado no rótulo da embalagem (na parte inferior do rótulo à esquerda, no lugar ou ao lado do código de barra). Esta empresa também vende o mesmo produto, porém sem selo ambiental, onde nenhuma parte das vendas é investida no meio ambiente. Além disso, algumas empresas produzem apenas produtos com selo ambiental e outras não tem selo ambiental.

Sua tarefa consiste em escolher, em várias situações, um entre dois produtos conforme os preços apresentados. Conforme a mudança de preço entre os dois produtos você deverá escolher um deles, marcando com um $\mathrm{X}$ ao lado da figura do produto escolhido. Observe atentamente os preços apresentados e faça uma nova escolha. Lembre-se que em cada par de produtos é uma nova escolha, independente das anteriores com novos preços. Em cada vez que forem apresentadas novas opções com novos produtos ou novos preços, tente escolher o mais próximo possível ao que escolheria se estivesse realizando a compra.

Se você tiver alguma dúvida, pergunte ao pesquisador. Ele ficará a sua disposição.

Ao final, chame o pesquisador.

Desde já agradecemos por sua participação. 
Aos participantes era apresentado um conjunto de pares de escolha entre cada um dos amaciantes, com preço médio de $\mathrm{R} \$ 10,00$ e a marca Comfort com selo de sustentabilidade fictício variando de $\mathrm{R} \$ 15,00$ a $\mathrm{R} \$ 5,00$ em 11 escolhas sucessivas e independentes, apresentadas em linha. Os participantes tinham que indicar sua escolha para todos os pares de preços apresentados e a média entre os preços com os quais ocorreu a mudança da escolha pela marca concorrente para o Comfort_Sustentável foi tomada como ponto de indiferença. Essas medidas de preço de indiferença foram relacionadas às medidas de Qualidade, Conhecimento e Sustentabilidade obtidas no Estudo 2 de Pedroso (2016).

\section{Resultados}

\section{Experimento 1}

Na Figura 1 estão apresentados os preços de indiferença relativos para cada um dos produtos, (Amaciante de Roupas, Lâmpada, Cama e Papel Sulfite). O cálculo dessa medida foi realizado a partir da média do preço com o qual os participantes mudaram suas escolhas pela marca com preço variável para a marca com preço fixo, dividido pelo preço médio de cada tipo de produto, nas condições com instrução genérica e com instrução sustentável (ou pró-ambiental).

Para o produto amaciante, os preços de indiferença relativos ficaram em 0,92 e 1,01 nas condições com instrução genérica e sustentável, respectivamente, indicando que com instrução genérica o preço pago pelo produto não sustentável ficou em $\mathrm{R} \$ 9,20$, abaixo do preço base do produto sustentável $(\mathrm{R} \$ 10,00)$ e na condição sustentável os preços relativos dos dois produtos ficaram semelhantes.

Para a Lâmpada os dois preços de indiferença foram de 2,64 com instrução genérica e 2,66 com instrução sustentável, de forma que o preço pago pela lâmpada com o selo energy saver foi aproximadamente $\mathrm{R} \$ 13,20$, maior que o da lâmpada halógena, fixo a $\mathrm{R} \$ 5,00$ nas duas condições. Cabe ressaltar que o valor máximo da lâmpada com energy saver no protocolo de escolha era de $\mathrm{R} \$ 15,00$ e que 18 dos 24 participantes apresentaram preferência exclusiva pela lâmpada halógena, indicando que o preço que pagariam pelo produto sustentável, nas duas instruções, poderia ser ainda maior que o especificado na coleta.

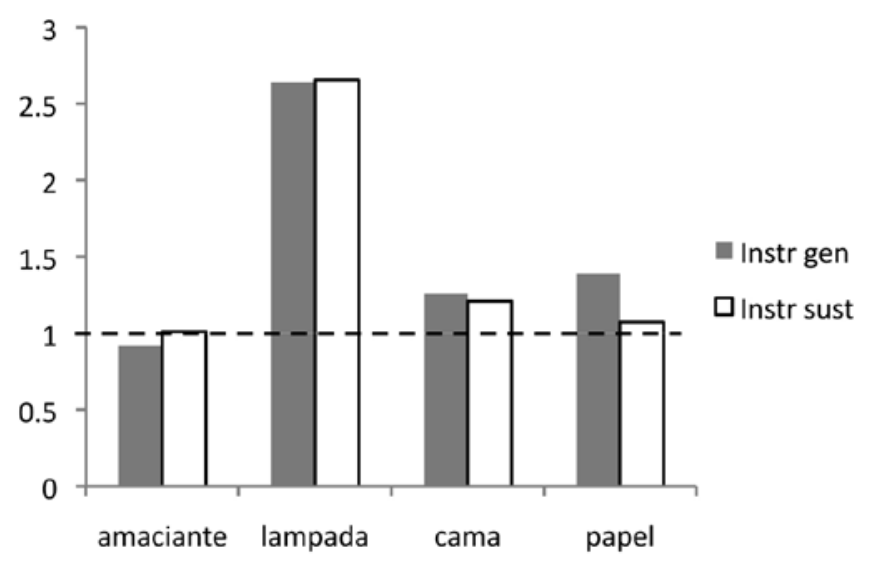

Figura 1 - Ponto de indiferença relativo ao valor total da marca com preço fixo para os produtos Amaciante, Lâmpada, Cama e Papel Sulfite, nas condições com instrução genérica e instrução sustentável. A linha tracejada indica o valor relativo com o qual o preço pago aos dois produtos é o mesmo. Para amaciante, dados abaixo da linha indicam maior preço do produto sustentável; para os demais produtos, dados acima da linha indicam maior preço para o sustentável.

As escolhas entre as camas e os papeis sulfite indicaram preços de indiferença relativos dos produtos sustentáveis maiores que os preços fixos dos produtos não sustentáveis. Nas duas situações, o preço com instrução genérica ficou acima do preço com instrução sustentável. Para cama, os preços relativos foram de 1,26, e 1,21 e para papel foram de 1,39 e 1,07, com instrução genérica e com instrução sustentável, respectivamente. Como o preço que variou nas escolhas foi o do produto sustentável para cama e papel sulfite, esses preços relativos obtidos indicam que os preços de indiferença da cama sustentável foram de $\mathrm{R} \$ 630,00$ e $\mathrm{R} \$ 605,00$ nas condições genérica e sustentável, em comparação à cama sem indicação de sustentabilidade $(\mathrm{R} \$ 500,00)$, e do papel reciclado foi de $\mathrm{R} \$ 34,75$ e $\mathrm{R} \$ 26,75$, frente ao papel não reciclado a $\mathrm{R} \$ 25,00$.

Para verificar se a diferença entre os preços era significativa, foi realizado o Teste $\mathrm{T}$ para amostras independentes, comparando-se os preços de indife- 
rença nas duas condições de instrução. Essa análise mostrou que os preços relativos foram semelhantes para os produtos Amaciante $(\mathrm{T}=-0,80, \mathrm{p}=0,22)$, Lâmpada ( $\mathrm{T}=-0,06, \mathrm{p}=0,48)$ e Cama $(\mathrm{T}=0,19$, $\mathrm{p}=0,43)$. Apenas nas escolhas com papel sulfite os valores na condição genérica foram significativamente maiores que na condição com instrução sustentável $(\mathrm{T}=1,79, \mathrm{p}=0,04)$.

Partindo-se da ausência de diferenças significativas entre as duas instruções para três dos quatro produtos, foi feita uma comparação das respostas de homens e mulheres, independentemente da condição, para amaciante, lâmpada e cama, a partir do Teste T. A análise indicou que o preço de indiferença médio das participantes do sexo feminino foi significativamente maior para o produto amaciante $(\mathrm{T}=3,63, \mathrm{p}=0,002)$, não sendo observada diferença para nenhum dos outros dois produtos.

Para que fosse verificado se participantes com diferentes características teriam padrões diferenciados de escolha, os preços de indiferença para cada produto foram correlacionados separadamente com idade e renda. As análises mostraram que para os dois casos e com os quatro produtos utilizados, não foram obtidas correlações significativas. Para idade, as correlações obtidas foram de $-0,03,0,13$, 0,39 e -0,35, com amaciante, lâmpada, cama e papel sulfite, respectivamente. Para renda, as correlações foram de $-0,15$ para amaciante, 0,12 para lâmpada, 0,17 para cama e 0,19 para papel.

Mesmo que os dados apresentados na Figura 1 demonstrem que para os produtos lâmpada, cama e papel tenham sido obtidos valores maiores para o produto sustentável nas condições com e sem instrução (exceto para papel na condição genérica, sendo o valor maior para o produto não sustentável), ou seja, o preço obtido foi maior que o preço esperado de cada valor para o produto sustentável, a diferença foi significativa (Dados obtidos a partir do teste Wilcoxon.) para o produto lâmpada (p. 002 e z. 3.06 com instrução genérica e sustentável). Para papel, a diferença foi significativa na condição genérica, mas para o produto não sustentável (papel p = 0.03 e $\mathrm{z}=2.12$ com instrução genérica; $\mathrm{p}=0,35 \mathrm{e} \mathrm{z}$ $=0.94$ com instrução sustentável). O produto cama não demonstrou diferença significativa para as duas condições de instrução (cama $\mathrm{p}=0.12$ e z 1.57 para instrução genérica; $\mathrm{p}=.027$ e $\mathrm{z}=1.10)$.

\section{Experimento 2}

A análise tem como foco principal os preços de indiferença entre o amaciante com selo sustentável e o amaciante sem o selo sustentável nos quatro conjuntos de escolha, além da relação do preço de indiferença e os níveis relativos de Conhecimento, Qualidade e Sustentabilidade, conforme obtidos no Experimento 2 do presente trabalho. Para tanto, foram utilizados os preços de indiferença obtidos nas escolhas entre os amaciantes de diferentes marcas sustentáveis (Ypê e Comfort_sustentável modificado experimentalmente) e não sustentáveis (Comfort, Monbijou e Babysoft). Para os níveis de conhecimento, qualidade e sustentabilidade, os dados obtidos no Experimento 2 foram relativizados entre as marcas em cada situação de escolha. Quando, por exemplo, a escolha foi entre Ypê e Comfort, a medida de Qualidade do Amaciante Ypê $(2,48)$ foi dividida pela do Comfort $(2,58)$, gerando uma qualidade relativa de 0,96 . A mesma sistemática foi utilizada para as demais escolhas e para as demais variáveis.

A Figura 2 apresenta os preços de indiferença entre os quatro pares de amaciantes, como função dos níveis relativos de conhecimento, qualidade, MCQ, sustentabilidade e MCQ_Sust, que acrescenta a Sustentabilidade como um critério de diferença relativa entre os produtos. As linhas referem-se às respectivas tendências lineares com melhores ajustes obtidas em regressão simples.

Para conhecimento, qualidade e MCQ relativos ao respectivo nível do amaciante Comfort, observa-se uma tendência de redução do preço de indiferença com o aumento do nível do produto alternativo relativamente ao amaciante Comfort, com um ajuste (R2) de 0,53 para conhecimento, 0,47 para qualidade e 0,5 para o MCQ, que envolve as duas variáveis anteriores. Os valores de inclinação que variaram de 13,36 para MCQ a 14,57 para conhecimento sinalizam alterações substanciais no preço de indiferença com leves reduções na diferença entre os respectivos níveis.

O preço de indiferença não se mostrou sensível às diferenças no fator sustentabilidade. $\mathrm{O}$ coeficiente de regressão obtido nesta análise ficou em 0,09 , relacionado ao fato de que tanto valores de 
sustentabilidade relativos baixos quanto altos foram acompanhados de preços de indiferença maiores ou menores. A incorporação da sustentabilidade ao MCQ também gerou um coeficiente de determinação baixo sobre os preços de indiferença, com um R2 de 0,32, abaixo do obtido apenas com o MCQ.

As análises acima mostram que o MCQ isoladamente foi mais determinante nos preços de indiferença que a sustentabilidade ou a utilização desta medida para uma nova média que gerou o MCQ
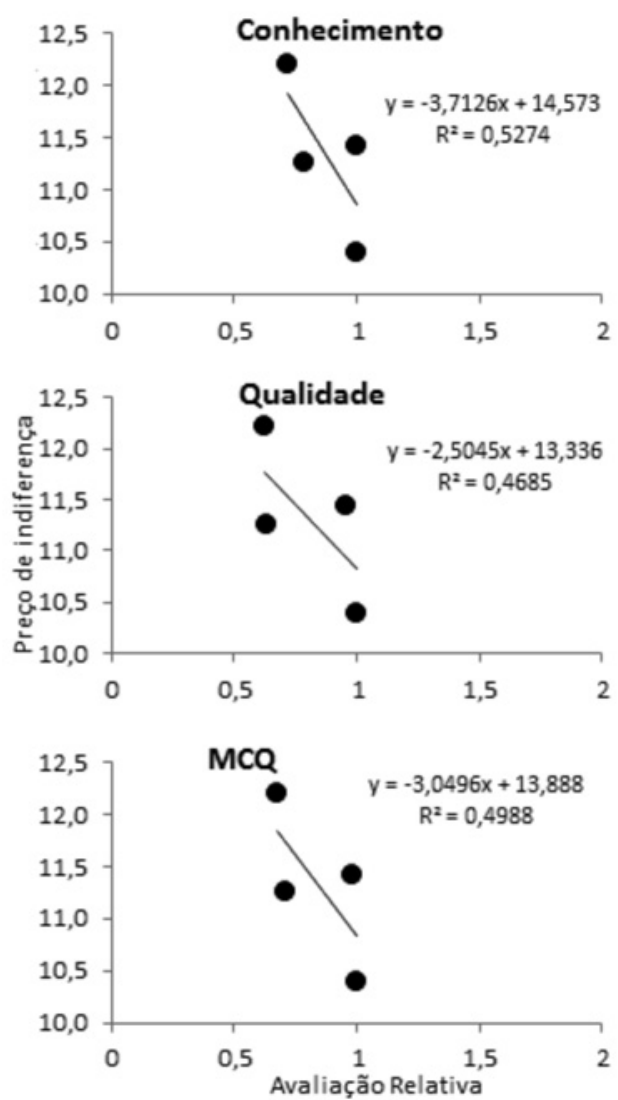

sust. Isso pode indicar falta de controle pela sustentabilidade ou uma interação dessas medidas na definição dos preços. Para investigar essa questão, foi realizada uma regressão múltipla tendo como variáveis independentes o MCQ e sustentabilidade. O R2 múltiplo ajustado obtido nesta análise foi de 0,59 , com os coeficientes de $-4,12$ para MCQ e 2,86 para sustentabilidade, indicando maior determinação pelo MCQ e direções opostas das duas variáveis nas suas influências sobre o preço de equivalência.
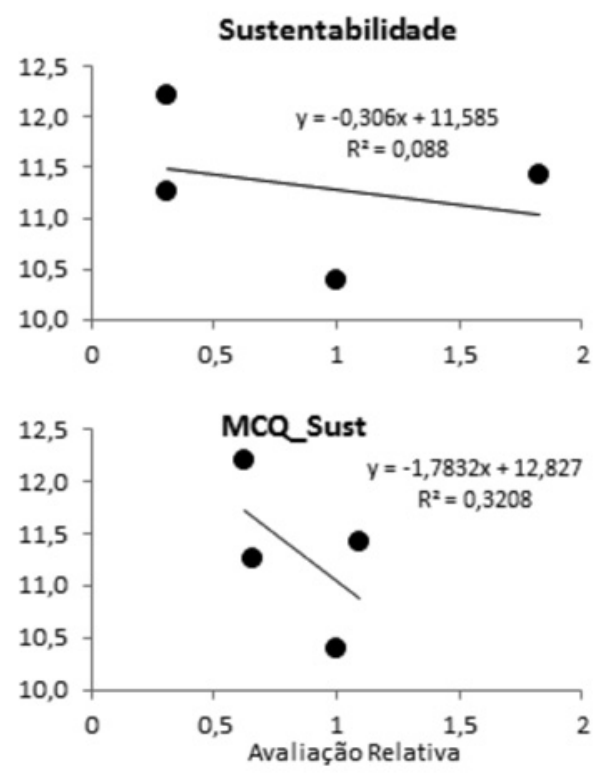

Figura 2 - Preço de indiferença (valor subjetivo) como função do nível de conhecimento, qualidade, sustentabilidade, Média de Conhecimento e Qualidade, e MCQ_Sust, que incorpora sustentabilidade à média de Conhecimento e Qualidade.

\section{Discussão}

Os dados do Experimento 1 mostraram que os preços de indiferença relativos das marcas com selo ambiental foram semelhantes ou maiores que as das marcas sem selo ambiental, principalmente para o produto lâmpada. Apesar disso, a instrução chamando a atenção para as consequências da utilização dos recursos naturais só teve efeito signifi- cativo com papel sulfite e oposto ao esperado: com instrução genérica, o preço do papel não reciclado foi maior que do reciclado. Esse efeito pode ser derivado do próprio leiaute das embalagens de papel utilizadas. O papel reciclado, com o selo ABNT ISO14000 pode ter evocado conhecimentos referentes a normas técnicas que não estão relaciona- 
dos a aspectos de sustentabilidade. Esta suposição é corroborada pelos dados do Experimento 1 do presente trabalho, no qual a maioria dos participantes, também pertencentes ao contexto universitário, indicou que o selo significava normas técnicas (de trabalhos acadêmicos). Já o papel não reciclado, apesar de não apresentar o mesmo selo, continha o selo CEFLOR, de reflorestamento.

Como não foi indicado aos participantes qual dos dois produtos estava sendo considerado sustentável, possivelmente os aspectos gráficos exerceram controles diferentes dos programados pelo fabricante e pela empresa responsável pelas características físicas do selo. Essa interpretação vai ao encontro da noção de sombreamento (shadowing, Catania, 1999), segundo a qual a presença de um estímulo mais intenso (o nome ABNT em tipografia grande) impede ou reduz o controle por outros estímulos (ISO14000 em tipografia menor) ou bloqueio (blocking, Catania, 1999), no qual a história de condicionamento com um estímulo pode impedir o condicionamento com um estímulo novo.

O preço de indiferença relativo da lâmpada halógena, próximo ao máximo utilizado no protocolo fornece dados que apontam para a possibilidade de que o controle é exercido não pelo aspecto sustentável, mas por redução de consequências aversivas, no caso, a economia na conta de energia. Políticas Públicas de retirada gradual das lâmpadas incandescentes focaram, ao longo dos últimos anos, a equivalência funcional entre os dois produtos e a redução do gasto pessoal com energia. Com a entrada das lâmpadas de LED, uma avaliação do preço pago a esta em relação à halógena que possa vir a ser comparada em um futuro próximo pode indicar novas variações e preços de indiferenças controlados pela redução de gasto energético.

É importante sinalizar que tanto renda quanto idade não se correlacionaram com os preços de indiferença para nenhum dos produtos. Este dado sugere que há semelhança nas interações com a sustentabilidade dos participantes de diferentes idades, indicando que mesmo os mais novos ou com maior renda não deram valor significativamente maior aos produtos sustentáveis.

Segundo Foxall et al. (2004), consumidores que estão sob controle de produtos com alto ní- vel de reforçadores utilitários e informativos tenderiam a sofrer menor influência da variação de preço das marcas preferidas quando comparados a consumidores que compram produtos mais básicos (baixo nível de reforço utilitário e informativo). Dado que apenas pequena parcela da população tem condições financeiras para arcar com esse perfil de compras, não se pode esperar que a maioria das pessoas deixe de maximizar aspectos financeiros ao escolher uma marca não sustentável que tenha valor inferior a uma marca sustentável. A partir disso, apesar das variações de renda no presente estudo, a presença de apenas um participante com renda acima de $\mathrm{R} \$ 10.000,00$ sugere que os participantes de forma geral interajam de forma semelhante quando confrontados com o conflito preço a pagar e sustentabilidade.

Ao se analisar escolhas entre diferentes marcas de um determinado produto, os dados podem indicar efeitos da marca ou do nível de sustentabilidade, e separar os efeitos da marca e da sustentabilidade pode ser difícil. Para avaliar essas diferenças, escolhas tais como as realizadas no Experimento 2, entre marcas de um mesmo produto com diferentes níveis de conhecimento podem fornecer dados que permitam se estimar o quanto os preços de indiferença se relacionam ao nível de qualidade e conhecimento ou de sustentabilidade.

O Experimento 2 mostrou que o MCQ foi a variável determinante das escolhas e, consequentemente, dos preços de indiferença dos amaciantes utilizados neste estudo. As variações do preço de indiferença se mostraram consistentes com dados anteriores que relacionaram MCQ, preço e consumo (Faustino, 2009; Silva, 2007). Contudo, o maior nível de determinação obtido no presente trabalho, de 0,52 para Conhecimento, foi menor que nesses estudos. Uma possibilidade reside no fato de que em estudos utilizando MCQ, os participantes realizaram escolhas e também responderam sobre o MCQ. Já no presente estudo, o MCQ foi obtido do Experimento 2 de Pedroso (2016), com participantes diferentes, apesar de terem sido retirados da mesma amostra. Um estudo posterior deve, para investigar essa questão, estimar o MCQ e os preços de indiferença, possivelmente vinculando-os a situação de compra real com os mesmos indivíduos. 
Os dados do Experimento 2 indicam ainda que sustentabilidade não gerou acréscimo de valor informativo ou utilitário às marcas que apresentavam selo de certificação. Essa suposição é corroborada pela semelhança no preço de indiferença do amaciante Comfort_sustentável (modificado experimentalmente), que foi de $\mathrm{R} \$ 10,39$, ou seja, praticamente o preço-base do produto sem apresentação de selo ambiental.

Pode-se supor, pelos dados de variação de preço relativo em função do nível relativo de sustentabilidade do presente estudo, que em condições discriminativas semelhantes (tamanho do selo, por exemplo) que se manteria o controle pelas mesmas variáveis apontadas por Foxall et al. (2006). Cabe ressaltar que o MCQ tem se mostrado promissor na estimativa de níveis de reforços informativos e sua relação com consumo em diferentes situações (Dias \& Oliveira-Castro, 2006; Faustino, 2009, Pohl \& Oliveira-Castro, 2004).

No presente estudo, os preços de um dos produtos variaram, diferentemente do que nos confrontamos em supermercado. Esta proposta consistiu de uma tentativa de estender a metodologia utilizada em escolhas com atraso e probabilidade para a escolha entre produtos e marcas que apresentam diferenças em um dos atributos, mantendo-se fixo o preço de uma das marcas e variando-se o preço da marca alternativa. Apesar de isto já ter sido feito com dados mostrando relação entre MCQ e preço de equivalência (Silva, 2007), o presente trabalho incorporou a alteração das propriedades de uma das marcas (inserção do selo ambiental na foto de uma das marcas). Com isso, foram obtidos preços para os quais se supõe que os dois produtos se igualam em relação a seus níveis de qualidade, conhecimento, preços e sustentabilidade. A ausência de relação entre preço e sustentabilidade mostra que a presença do selo aparentemente não teve propriedades discriminativas. Isso indica que essa metodologia pode ser efetiva em manipular, em novos estudos, um conjunto maior de variáveis, como o tamanho/posição do selo.

\section{Referências}

Back, L., Hahn, I. S., \& Scherer, F. L. (2015). A Consciência ambiental e as atitudes de consumo sustentável de estrangeiros. Reunir Revista de Administração, Contabilidade e Sustentabilidade, 5(2), 25-42. doi: 10.18696/ reunir.v5i2.259

Barbosa, M. L. A., Araújo, E. F. M., \& Cabral, S. B. C. (2014). Rótulos ambientais como argumento de sustentabilidade e suas relações com a intenção de compra de serviços hoteleiros. Anais do XI Seminário da Associação Nacional Pesquisa e Pós-Graduação em Turismo, Universidade do Estado do Ceará-UECE, Fortaleza, CE, Brasil, 1490. Disponível em: https://www.anptur.org. br/anais/anais/sumario.php?versao $=11$

Baum, W. N. (1974). On two types of deviation from matching: Bias and undermatching. Journal of the Experimental Analysis of Behavior, 22, 23142. doi: 10.1901/jeab.1974.22-231

Botelho, J. C. B., \& Manolescu, F. M. K. (2010). $\mathrm{O}$ marketing verde como diferencial competitivo nas organizações. In: XIV Encontro Latino Americano de Iniciação Científica e $X$ Encontro Latino Americano de Pós-Graduação - Universidade do Vale do Paraíba, São José dos Campos, SP, Brasil, 1. Disponível em http:// www.inicepg.univap.br/cd/INIC_2010/anais/ arquivos/0003_0495_01.pdf

Braga, M. V. N., de Albuquerque, L. C., \& Paracampo, C. C. P. (2005). Análise dos efeitos de perguntas e de instruções sobre o comportamento não-verbal. Interação em Psicologia, 9(1), 77-89 . doi: 10.5380/psi.v9i1.3288

Braga, R. C. M. S., Viana, j. F. C., Abreu, L. M., \& Faria, R. C. (2008). Valoração ambiental de áreas protegidas para a gestão ambiental. OLAM Ciência \& Tecnologia, 8(1), 68-87.

Campos, L. M. S., Grzebeiluckas, C., \& Selig, P. M. (2009). As empresas com certificação ISO 14001 são mais rentáveis? Uma abordagem em companhias abertas no Brasil. Revista Eletrônica de Administração, 15(1), 08-131.

Castro, J. L. C., Paracampo, C. C. P., \& Albuquerque, L. C. (2015). Efeitos de justificativas e de consequências imediatas sobre o comportamento 
de seguir regras. Acta Comportamentalia, 23(2), 153-166.

Catania, C. A. (1999). Aprendizagem: comportamento, linguagem e cognição. Porto Alegre: Artmed.

Cavalcanti, P. R., Oliveira-Castro, J. M., \& Foxall, G. R. (2013). Individual differences in consumer buying patterns: a behavioral economic analysis. The Psychological Record,63(2), 259-276. doi: 10.11133/j.tpr.2013.63.2.003

Coelho, C., Hanna, E. S., \& Todorov, J. C. (2003). Magnitude, atraso e probabilidade de reforço em situações hipotéticas de risco. Psicologia: Teoria e Pesquisa, 19(3), 269-278. doi: 10.1590/ S0102-37722003000300009.

De Toni, D., Mattia, A., \& Larentis, F. (2012). Consumo consciente, valor e lealdade em produtos ecologicamente corretos. Revista de Administração FACES Journal, 11(3), 136-156. doi : 10.21714/1984-6975FACES2012V11N3ART975

Deus, N. S., Felizola, M. P. M., \& Silva, C. E. (2010). O consumidor socioambiental e seu comportamento frente aos selos de produtos responsáveis. Revista Brasileira de Administração Científica, 1(1), 32-54. doi: 10.6008/ ESS2179-684X.2010.001.0002

Dias, G. M. M. (2008). Qual o critério da rotulagem ambiental? Revista em Agronegócio e Meio Ambiente, 1(1), 279-289.

Dias, M. B., \& Oliveira-Castro, J. (2006). Comportamento de procura por produtos: efeitos da quantidade de marcas. Revista Psicologia Organizacional e do Trabalho, 6(1), 195-232.

Fabi, M. J. S., Lourenço, C. D. S., \& Silva, S. S. (2010). Consumo consciente: a atitude do cliente perante o comportamento socioambiental empresarial. In Anais do IV Encontro de Marketing da ANPAD, Florianópolis, SC, Brasil, 1. Recuperado de http://www.anpad.org.br/ad$\mathrm{min} / \mathrm{pdf} / \mathrm{ema} 51 . \mathrm{pdf}$

Faustino, R. C. (2009). Estimativa de qualidade, conhecimento e valor de atributos utilitários de veículos. Dissertação, Mestrado em Psicologia, Pontifícia Universidade Católica de Goiás, Goiânia, GO.

Félix, J. D. B., \& Nunes, A. (2014). A disposição a pagar pela sustentabilidade ambiental. Desenvolvimento em Debate, 3(1), 111-123.
Recuperado de http://desenvolvimentoemdebate.ie.ufrj.br/pdf/dd_3_1_FELIX_E_NUNES.pdf

Foxall, G. R, Oliveria-Castro, J. M., James, V. K., Yani-de-Soriano, M. M., \& Sigurdsson, V. (2006). Consumer Behavior analysis and social marketing: the case of environmental conservation. Behavior and Social Issues, 15, 101-124. doi: 10.5210/bsi.v15i1.338

Foxall, G. R., Oliveira-Castro, J. M., \& Schrezenmaier, T. C. (2004). The behavioral economics of consumer brand choice: patterns of reinforcement and utility maximization. Behavioural Processes, 66(3), 235-260. doi: 10.1057/9780230596733_6

Foxall G. R., James, V. K., Oliveira-Castro, J. M. \& Ribier, S. (2010). Product Substitutability and the Matching Law. The Psychological Record, 60, 185-216. Doi: 10.1007/BF03395703

Furtado, C. (1974). O mito do desenvolvimento econômico. Rio de Janeiro: Paz e Terra.

Furtado, C. (2009). Desenvolvimento e subdesenvolvimento. Rio de Janeiro: Contraponto.

Godoy, A. M. G., \& Biazin, C. C. (2000). A rotulagem ambiental no comércio internacional. Anais $4^{\circ}$ Encontro Eco-Eco, disponível em www. ecoeco.org.br/conteudo/publicacoes/encontros/iv_en/.../2.pdf, em outubro de 2015.

Gomes, D. V. (2006). Educação para o consumo ético e sustentável. Revista Eletrônica do Mestrado em Educação Ambiental, 16, 18-31. doi: 10.14295/remea.v16i0.2778

Gonzaga, C. A. M. (2005). Marketing verde de produtos florestais: teoria e prática. Floresta, 35(2). doi: 10.5380/rf.v35i2.4623

Gorni, P. M., Gomes, G., \& Dreher, M. T. (2012). Consciência ambiental e gênero: os universitários e o Consumo sustentável. Revista de Gestão Social e Ambiental, 6(2), 165-179. doi: 10.24857/rgsa.v6i2.468

Herrnstein, R. (1961). Relative and absolute strength of response as a function of frequency of reinforcement. Journal of the Experimental Analysis of Behavior, 4, 267-272. doi: 10.1901/ jeab.1961.4-267

Herrnstein, R. J. (1970). On the law of effect. Journal of the Experimental Analysis of Behavior, 13, 243-266. doi: 10.1901/jeab.1970.13-243/

Hirsh, J. L., Costello, M. S., \& Fuqua, R. W. (2015). 
Analysis of delay discounting as a psychological measure of sustainable behavior. Behavior and Social Issues, 24, 187-202. doi: 10.5210/bsi. v24i0.5906

Kohlrausch, A. K. (2003). A rotulagem ambiental no auxílio à formação de consumidores conscientes. Dissertação de Mestrado, Programa de Pós-Graduação em Engenharia de Produção, Universidade Federal de Santa Catarina, Florianópolis.

Kohlrausch, A. K., Campos, L. M. S., \& Selig, P. M. (2004). Selos ambientais: qual seu papel e influência no processo de compra de produtos orgânicos?. Anais do XXIV Encontro Nac. de Eng. de Produção, Florianópolis, SC, Brasil. (pp. 53715378). Disponível em: http://www.abepro.org. br/biblioteca/enegep2004_enegep1005_1415.pdf

Macedo, R. C. (2009). Economia ambiental aplicada: uma abordagem baseada em SIG para análise custo-benefício. Trabalho de conclusão de curso. Programa de Pós-Graduação. Instituto Nacional de Pesquisas Espaciais, São José dos Campos, SP.

Mazur, J. (1998). Choice with delayed and probabilistic reinforcers: effects of prereinforcer and postreinforcer stimuli. Journal of the Experimental Analysis of Behavior, 70, 253-265. doi: 10.1901/jeab.1998.70-253

Nalini, L. E. G., Cardoso, M. M., \& Cunha, S. R. (2013). Comportamento do consumidor: uma introdução ao Behavioral Perspective Model (BPM). Fragmentos de Cultura, 23(4), 489-505. doi: 10.18224/frag.v23i4.2976

Oliveira-Castro, J. M., Foxall, G. R., \& Wells, V. K. (2010). Consumer brand choice: money allocation as a function of brand reinforcing attributes. Journal of Organizational Behavior Management, 30 (2), 161-175. doi: 10.1080/01608061003756455

Oliveira-Castro, J. M., Foxall, G. R., \& James, V. K. (2008). Individual differences in price responsiveness within and across brands. Services Industries Journal, 28(6), 733-753. doi: 10.1080/02642060801988605

Oliveira-castro, M., Foxall, G. R., \& Schrezenmaier, T. C. (2006). ConsumeI brand choice: Individuai and group analyses of demand elasticity. Journal of the Experimental Analysis of Behavior, 85(2), 147-166. doi: 10.1901/jeab.2006.51-04
Pedroso, R. (2016). Valor subjetivo do consumo sustentável. Tese de Doutorado. Pontifícia Universidade Católica de Goiás, Goiânia, GO.

Pinto, S. A. K., \& Pedroso, R. (2015). Desenvolvimento sustentável: o valor do consumo sustentável. Monografia. Curso de Psicologia. Faculdades Associadas de Ariquemes, Ariquemes, RO.

Pohl, R. H. B. F., \& Oliveira-Castro, J. M. (2004). Efeitos do nível de reforço informativo das marcas sobre a duração do comportamento de procura. Dissertação. Mestrado em Psicologia, Universidade de Brasília, Brasília, DF.

Rachlin, H., Raineri, A., \& Cross, D. (1991). Subjective probability and delay. Journal of the Experimental Analysis of Behavior, 55, 233-244. doi: 10.1901/jeab.1991.55-233

Rodrigues, A. R., Gonçalves, E. J. V., Costa, A. P., Nora, E. S., \& Rezende, D. C. (2011). Marketing Verde e Consumo consciente: segmentando o mercado de Lavras - MG. Anais do XXXV EnANPAD, Rio de Janeiro, RJ, Brasil, 1. Recuperado de http://www.anpad.org.br/ad$\mathrm{min} / \mathrm{pdf} / \mathrm{MKT} 2880 . \mathrm{pdf}$

Romeiro, A. R., \& Maia, A. G. (2011). Avaliação de custos e benefícios ambientais. Cadernos ENAP, 35. Brasília. Recuperado de http://repositorio. enap.gov.br/handle/1/657

Sachs, I. (2009). Caminhos para o desenvolvimento sustentável. Rio de Janeiro: Garamond.

Silva, M. E., \& Gómez, C. R. P. (2010). Consumo consciente: o papel contributivo da educação. Revista Reuna, 15(3), 43-54.

Silva, M. S. (2007). Análise do comportamento do consumidor: valor de equivalência e avaliação de qualidade e conhecimento de marcas de café. Dissertação. Mestrado em Psicologia, Pontifícia Universidade Católica de Goiás, Goiânia, GO.

Simonassi, L. E. (1999). Cognição: Contato com contingências e regras. Revista Brasileira de Terapia Comportamental e Cognitiva, 1(1), 8393. doi: 10.31505/rbtcc.v1i1.274

Skinner, B. F. (2003). Ciência e comportamento humano (J. C. Todorov \& R. Azzi, Trads.). São Paulo: Martins fontes. (Trabalho original publicado em 1953)

The Earth Charter Initiative (2003). A carta da terra. Disponível em: <www.earthcharter.org > Acesso em: 17/03/2017. 
Todorov, J. C. (2005). Laws and the complex control of behavior. Behavior and Social Issues, 14, 8690. doi: 10.5210/bsi.v14i2.360

Tomé, F. (2008). Tudo o que você precisa saber sobre selo verde e ecoetiquetas. São Paulo: Projeto W3. Disponível em: <http://www.musa.org.br/file. asp?id=50\&arquivo=ebook\&dir=livros\&titulo $=\mathrm{O} \% 20$ Selo $\% 20$ Verde $\% 20 \mathrm{e} \% 20$ Ecotiquetas. $>$. Acesso em setembro de 2015.

Tversky, A., \& Kahneman, D. (1981). The framing of decisions and the psychology of choice. Science, 211(4481), 453-458. doi: 10.1126/science. 7455683

Ulbra - Universidade Luterana do Brasil (2008). Microeconomia, 23(2), 153-166. Curitiba: Ibpex. Valent, J. Z., Vieira, T. R., Bruzza, A., Rodrigues, R. G. Celia, A. P., \& Schmidt, V. (2014). Fatores determinantes do consumo de alimentos certificados no brasil. Revista Eletrônica em Gestão, Educação e Tecnologia Ambiental-REGET, 18, 57-65. (Ed. Especial) doi: http://dx.doi. org/10.5902/2236117013094

Veras, M. A., Souza, J, Araújo, J. B. Filgueira, P. V. S., \& Silva, R. G. (2014). Análise sobre o consumo de produtos orgânicos com rótulos ambientais tipo I em Natal, RN. Anais do V Congresso Brasileiro de Gestão Ambiental, Belo Horizonte, MG. Disponivel em: https://www.ibeas.org.br/ congresso/Trabalhos2014/V-055.pdf

\section{Informações do Artigo}

Histórico do artigo:

Submetido em: 25/04/2018

Primeira decisão editorial: 06/05/2019

Versão definitiva aceita em: 14/06/2019

Editor Associado: Denis Roberto Zamignani 